\title{
Implicaciones internacionales del deterioro ambiental
}

\begin{abstract}
LUCIANO TOMASIN T, fue profesor de la Universidad de Chile y de la Universiclad Católica de Santiago y, posteriormente, Baring Research Fellow de la London School of Economics $\mathrm{y}$ el Royal Institute of Inlernational Affairs. Fue tambicin jefe de la Unidad de Desarrollo Social del BID, y' actualmente forma parte del Instituto para la Integración de América Latina. Ha publicado diversos trabajos en Estudios Internacionales, Revista Latinoamericana de Ciencia Politica, The World Today y otras rcvistas especializadas.
\end{abstract}

UNA VERSIÓN ECOLÓGICA DE LOS HORRORES DEL JUJCIO FINAL

"Cuando las gentes morian en tal cantidad que no había sitio suficiente para enterrarla en tierra consagrada, se cavaban en el interior de los cementerios grandes y profundas fosas comunes, donde los cadáveres eran arrojados como mercancla en un navio; cuando las fosas estaban llenas hasta el borde, las tapaban con una ligera capa de tierra". Así describia Boccaccio en el primer capítulo del Decamerón los estragos de la Peste Negra, que estalló en Europa en 1348 y que en menos de dos años segó una tercera parte de la población del viejo continente, al explicar los motivos que indujeron a una serie de nobles damas y señores a reunirse en un apacible castillo de la campiña florentina. Fue esa una época en que las lamentaciones se escuchaban por todas partes $y^{\prime}$ en que los profetas del desastre proliferaban. Sin embargo, en medio de tanta muerte $y$ desolación, estaba despuntando el Renacimiento.

Del mismo modo, tecnócratas y hombres de ciencia se reúnen en estos días en Bellagio, sobre el lago Como, o en Founex, junto

* E1 autor agradece a Eduardo Novaes y a Osvaldo Sunkel por las breves pero medulares criticas formuladas al borrador inicial de este trabajo. También es deudor de la reciente pero rica bibliografia referente al tema. Tomando en cuenta la existencia de numerosas compilaciones bibliográficas sobre el medio humano, en el trabajo sólo se citan las fuentes que fueron directamente utilizadas en el texto. 
al de Ginebra, para especular sobre el porvenir de la humanidad amenazado por la progresiva y acelerada destrucción del ecosistema natural del hombre. En los países avanzados se conienza a pensar con alarma que el crecimiento exponencial de la población, la industrialización y el consumo conducirán, en un plazo no muy distante, a un verdadero derrumbe económico y demográfico, ya sea como consecuencia del agotamiento de los recursos naturales, de la insuficiencia de alimentos o del aumento de la contaminación ambiental, o mejor aún, de una combinación de todos estos factores.

Es interesante observar cómo esta preocupación se expresa igualmente en movimientos de protesta y en círculos oficiales. Estudiantes venidos de todas partes de los Estados Unidos para protestar contra la guerra en Viet Nam sobre los prados que rodean el monumento de Washington, después de desafiar a la policía se organizan espontáneamente para recoger y quemar los desperdicios arrojados durante la manifestación, con el lin de preservar la pureza del paisaje, mientras que desde su alto sitial el Secretario General de las Naciones Unidas convoca una Conferencia Mundial sobre el Medio Humano en Estocolmo, Suecia.

Una expresión sintomática de esta preocupación son los trabajos del Club de Roma. El Club de Roma se gestó en abril de 1968, cuando un grupo de economistas, científicos, intelectuales y hombres públicos de todo el mundo se reunieron en la Accademia dei Lincei, por iniciativa del empresario italiano Dr. Aurelio Peccei, con el objeto de analizar la "problemática mundial", como ellos gustan llamar a las conflictivas tendencias de nuestro tiempo. El Club de Roma, una organización informal que ha sido descrita por alguien como una "universidad invisible", hoy cuenta con setenta miembros de veinticinco nacionalidades $y$ ya ha dado a la publicidad sus primeras conclusiones. ${ }^{1}$

Las actividades del Club de Roma se han concentrado en el Proyecto sobre la Situación de la Humanidad. Para desarrollarlo se empleó un modelo matemático, construido especialmente para analizar la evolución de las cinco tendencias principales del actual panorama mundial: el rápido incremento de la población, la desnutrición generalizada, el crecimiento de la industrialización, el agotamiento de los recursos naturales y la contaminación del medio ambiente. El modelo se basa en la información disponible hasta la fecha, e investiga el comportamiento de cada una de estas variables y sus interrelaciones, en extensos periodos de tiempo que

'Ver The Limits to Growth, A Report for the Club of Rome's Project on the Predicament of the Mankind, por D. H. Meadows et al. Universe Books, Nueva York, 1972. Para una evaluación similar de la amenaza ecológica, ver $A$ Blucprint for Survival en The Ecologist, vol. 2, No. 1, 1972. 
Ilegan hasta finales de la próxima centuria. Para hacerlo, naturalmente, se usó la computadora. Sus conclusiones no fueron optimistas.

Si las presentes tendencias en la población mundial, la industrialización, la contaminación, la producción de alimentos y el agotamiento de los recursos continúan sin cambio alguno, los límites del crecimiento posible en este planeta se alcanzarán en algún momento dentro de los próximos cien años. El resultado más probable será una declinación más bien repentina e incontrolable tanto en la población como en la capacidad industrial.?

La computadora procesó diversas alternativas que diferían entre sí en cuanto al comportamiento atribuido a una o más de las cinco variables consideradas en el modelo.

La mera extrapolación de las tendencias conocidas con respecto a cada una de las variables mencionadas permitió trazar el cuadro de un desarrollo industrial basado en una creciente utilización de recursos no renovables. La contaminación aumentaria. Para -obtener nuevos recursos, debido a su agotamiento y a la elevación de sus precios, sería necesario emplear cada vez más capital, reduciendo asi la inversión destinada a hacer posible el crecimiento futuro. Eventualmente, Ia inversión quedaría a la zaga de la depreciación y la base industrial de la sociedad se derrumbaria, arrastrando consigo la agricultura y los servicios, fuertemente orientados y'a hacia la producción de insumos industriales. Por último, la población misma comenzaria a disminuir como consecuencia del aumento de las tasas de mortalidad ocasionado por la falta de alimentos y de servicios de salubridad. El colapso se produce, pues, por agotamiento de los recursos no renovables acompañado de un exceso de contaminación ambiental y de falta de alimentos.

Hasta aquí no se han considerado las potencialidades que - ofrece el desarrollo tecnológico para enfrentar esos problemas. Al tomarlas en cuenta, sin embargo, los resultados no son mucho mejores.

- Una primera alternativa jucga con la posibilidad de que como consecuencia del desarrollo de la energía nuclear, la creciente utilización de minerales de baja ley y la explotación masiva del lecho marino la humanidad pueda disponer de recursos prácticamente ilimitados. En este caso, muestra la computadora, el crecimiento se detendría en un plazo no mucho más largo que en la hipótesis anterior, por el aumento inmoderado de la contaminación ambiental.

- Otra alternativa contempla la posibilidad de reducir esta

¿The Limits to Growlh, op. cit., p. 23. 
última mediante el "reciclaje" o rcaprovechamiento de los recursos naturales y la introclucción de tecnología especialmente diseñada para controlar la contaminación. Se toma cn cuenta, sin embargo, que el costo cle este control se eleva rápidamente conforme los standards ecológicos se vuclven más severos. Es asi como, por ejemplo, para pasar del $66 \%$ al $69 \%$ en la reclucción de partículas atmosféricas en una ciudad norteamericana es preciso elevar los costos de 7,5 millones de dólares a 26 millones. En este caso, con todo, el colapso sobreviene uno o dos decenios mís tarde por insuficiencia de alimentos.

- En una terccra hipótesis, los clectos combinados del aumento de la producción agrícola acarreado por la revolución verde y del control de la nataliclad perniten operar un modelo con recursos ilimitados, control de la contaminación y abundancia de alimentos. Lo que ocurre ell este caso es que la producción industrial y agricola por halbitante se expande de tal manera que la contaminación rompe todas las bareras tecnológicas, detenienclo el crecimiento antes del año 2100.

La conclusión a que se llega es que la aplicación de la tecnologia podria prolongar en cl tiempo el proceso de crecimiento, pero seríat incapaz de remover sus últimos límites. "Podemos - dice el Club de Roma- decir con cierta confianza (sic) que, si presumimos que el sistema actual no experimentará cambios mayores, el crecimiento de la población y la industrialización se cletendraín ciertamente dentro del próximo siglo, a lo menos"."

Los resultados que arroja este tipo de ejercicios son, como puede verse, alarnantes. Sin embargo, debemos guarclarnos bien de aceptar estos modelos por su valor nominal. Resulta aplicable a este caso lo que sucede frecuentemente con cicrtos temas y personas, a los cuales hay que comenzar por despojar de su solemnidad para poder tomarlos en scrio.

En su célebre Ensayo publicado en 1798, Malthus sostuvo que, mientras los recursos mundiales de alimentos cran limitados, no haloía límites potenciatcs al crecimiento de la población, la que en breve superaría los viveres disponibles. Sin embargo, el incremento clemográlico fue compensado con creces por los progresos agricolas realizados en Europa y por la incorporación cle nuevas tierras en América, Africa y Occanía. Como consecuencia cle la revolución ini-

3Para una critica a los modelos del Cluls de Rona, ver Helio Jaguaribe, El Equilibrio Ecológico Mundial y los Paises Subdesarrollaclos, en Estudios Intermacionales No 17 , encro-marto de 1972. 
ciada en los transportes por el cluque de Briclgewater en J759, cuanclo construyó un canal que le permitió vender a bajo precio su carbón en Manchester, hacia el año 1800 lnglatema disponia de S.000 Km. de agua navegables. Precisamente cuando alcanzaba los. limites para el aprovechamiento de sus aguas interiores, hacia 1820, comenzaron a establecerse los primeros ferrocarriles. Algo siinilar ocurrió con los esfuerzos cle los ingleses para cubrir la isla de encinas con el objeto de alimentar su poclerosa industria naval $y^{\prime}$ mantener el control de los mares. Todavia en la guerra de Grimea (1854-1856) la flota británical incluía una mezcla abigarrada de navios a vela y a vapor, sin un solo barco metálico, pero pocos años después la construcción de barcos de hierro tornaron obsoletas aquellas preocupaciones. Lo mismo calje observar acerca de la ansiedad de las potencias europeas, durante el siglo pasado, por el control del carbón, el cual pronto fue superado por el petróleo como fuente de energia. Hay' que reconocer que todavía hoy estos problemas son tratados a menudo con tonos apocalipticos, como cuando se especula con la fusión artificial de los casquetes polares, la remodelilción cle Siberia o la inundación del Amazonas.

Pero, habienclo despojado a las tesis del Club de Roma de una parte de sus ropajes majestuosos, no saltemos sin más a la conclusión de que "el emperador va desnudo".

\section{ALGUNOS INDICADORES DEL DETERIORO AMBIENTAL}

Los indicadores objetivos del cleterioro de la ecología son numerosos y clocuentes, particularmente en el caso cle la contaminación atmosférica. El $97 \%$ de la producción mundial de energía industrial provicne de la utilización de combustilules fósiles -carbón, petróleo $y^{*}$ gas natural- que producen al quemarse, entre otras. sustancias tóxicas, clióxido de carbono $\left(\mathrm{CO}_{2}\right)$. La combustión de estos clementos arroja anualmente en lat atmósfera alrededor de veinte mil millones de toneladas de $\mathrm{CO}_{2}$. A pesar de que solamente la mitad de esa masa róxica ha a parecido en la atmósfern, y de que la otral mitad ha siclo absorbicla aparentemente por la superficic marina, la presencia de $\mathrm{CO}_{2}$ ell la atmós [cra está crecienclo exponencialmente a una tasa de $0,2 \%$ anual. Un electo supletorio de la genernción de energía se expresa bajo la forma de la contaminación termal que provoca profundas perturbaciones en el equilibrio de la vida acuálica o islotes cle calor en torno de las grandes ciudacles. Debe tencrse presente que una clevación general de la temperatura terrestre de dos grados centigrados de magnitud bastaría para producir la fusión de los casquetes polares, lo que sc traduci- 
ría en profundas transformaciones climáticas $y^{\prime}$ en la elevación del nivel de los océanos en todo el planeta, con la subsiguiente desaparición de algunas grandes ciuclacies, especialmente en Europa Occidental. No debe desestimarse la acción del monóxido de carbono, cl dióxido de sulfuro y los óxidos de nitrógeno, así como del melcurio y cl plomo resultantes, segúm los casos, cle la producción de energía, los aviones y automotores, la industria química y eléctrica, 1a inclustria de la pulpa y el papel y de muchas otras actividades. Por otra parte, aún no se conoce la magnitud que adoptarí ni los electos que traerá consigo la contaminación nuclear derivada de la utilización industrial cle nucvis luentes de cnergía.

Similar es el cuadro que presentan los océanos. Se estima que cada año se mezclan con el mar aproximadlamente cinco millones de tonelaclas de petróleo, lo que equivale al $2 \%$ de la producción mundial, como consecuencia de operaciones vinculadas al transporte de hidrocarburos y a la explotación de reservals submarinas. En aquellas zonas en que es posible encontrarlo en mayor densidad, el petróleo destruye cl plancton marino, lo que afecta drásticamente la vida de los peces y aun la de los pájaros. Otra fuente importante de contaminación está constituida por los efluvios industriales $y$ privacios -compuestos estos últimos básicamente por detergentes y aguas servidas - así como por restos de fertilizantes. Estos elluvios son responsables en gran parte de la lenta asfixia que sc observa en algunos mares, entre los cuales el Báltico y el Adriático parecen ser los más afectados hasta ahora. La desoxigenación del agua se produce por la presencia de un exceso de elementos fertilizantes cn el medio marino, lo que provoca una proliferación anormal de ciertas especies y un consumo tambiéı anolmal de oxígeno. Una consecuencia visible de este fenómeno es la formación de "mares rojos", cubieltos de algas propensas a la descomposicion.

El suelo tampoco escapa al deterioro ecológico. Y'a se ha hecho mención a los efluvios tóxicos provenientes de las ciudades y los asentamientos humanos. A ello cabc añadir el creciente volumen de desperdicios sólidos generados por socicdades de alto consumo. La falta de previsión ha determinado que el mundo haya perdido ya 500 millones de hectarcas de tierra cultivable por los efectos combinados de la erosión $y$ la salinización. Descle otro punto de vista, cada dia se cobra más conciencia de la gravedad que alcanza el empobrecimiento de la tierra como consecuencia de la tala cle bosques y la habilitación de terrenos urbanos. La contaminación de las aguas interiores también ha alcanzado proporciones alarmantes, de lo cual es un buen ejemplo la situación de los Grandes Lagos en los Estados Uniclos o la ambigua función que clebe cumplir el Rin 
al servir de desaguadero a Francia y Alemania y de fuente de agua: potable para Holancla.

Las grandes obras de ingenieria realizaclas por el hombre gracias. a los poderosos recursos que la tecnologia contemporánea ha colocado en sus manos, son otras fuentes de alteración del equilibrio ecológico. Como ejemplo basta señalar la represa de Asuán, destinada a movilizar el esfuero del pueblo egipcio y a servir de testimonio de la modernización del país y de la amistad soviética frente a los pueblos árabes. I.a represa, cuya construcción comenzó en 1960, permitió la creación del lago artificial más grande del mundo, el cual contiene en la actualidiad 175 mil millones de metros cúbicos de agua, e incorporar alrededor de 800 mil hectáreas de nuevas tierras cultivables. Sin embargo, sus efectos ecológicos adversos comenzarou a apreciarse a comienzos de 1962, en forma de temblores de tierra que han proseguiclo inasta ahora. En 1972 se produjo un temblor en Kariba, una regrión africana en que ello jamás habia ocurriclo antes. El mismo tipo de fenómenos se ha producido en todo el mundo como consecuencia de la construcción de grancles embalses. La represa de Koyna, en la India, provocó en 1967 un sismo que arrojó un saldo de más de 200 muertos, y en Grecia después de la ampliación del lago artificial de Kremasta más de 500 casas fueron destruidas por un movimiento de tierra. Pero las secuelas ecológicas de la represa de Asuán no se limitan a estos aspectos, sino que comprenden también la ruptura del equilibrio biológico en extensás zonas alcdañas. Una de las consecuencias de este fenómeno es la trágicá dilusión de la esquitosomiasis por las zonas ruxales del país a raí de haberse reemplazado el sistema basado en las perióclicas inundaciones del Nilo por la irrigación artificial. La desaparición de las sardinas en cl Meditcrráneo es otra consecuencia directa cle la construcción del Asuán. Las sarclinas del Mediccráneo, un mar pobre en sustancias orgánicas, se nutrian principalmente de los ricos clepósitos de limo arrastrados por el Nilo. Antes cle que la represa entrara en operaciones, los pescadores egipcios recogían anualmente 18.000 Loneladas cle sardinas, mientras que hoy dia apenas alcanzan a pescar 500 tonelachas.

El conocimiento existente acerca de estos problemas no permite apreciar con ningún grado probable de exactitud los elcclos que podrían derivarse de la acción cle estos factorcs. No se conocen los límites de la capaciclacl de la tierra para absorber la contaminación. Tampoco es posible prever cuándo se alcanzarán esos límites, debido a que los agentes suelen producir sus efectos con considerable rctardo. Si a partir de 1970 hubiera comenzado a declinar el uso de DDT como consecuencia de la aplicación de políticas ambientales, su efecto sobre los peces continuaria aumentando por espacio de 
10 años, y no volvería a alcanzar los niveles correspondientes at 1970 antes de 1995. Por último, debe señalarse que con frecuencia la contaminación se transmite a escala global o regional. Es lo que ocurre, por ejemplo, con el bióxido de carbono: los Estados Unido: disponen dentro de sus fronteras nacionales de tan solo el $60 \%$ del oxígeno que consumen. En una región tan alejada de los grandes centros industriales como es Groenlandia, los residuos de plomo han aumentado en un $300 \%$ desde 1940. La Península Escandinava se ve obligada a absorber grandes cantidades de sustancias ácidas, que interfieren con la pesca tan importante para la región, originadas en las emisiones de óxido de sulfuro que producen en Ias. zonas industriales de Alemania y Gran Bretaña. Del mismo modo, en Europa se han observado acumulaciones de humo provenientes de incendios de bosques en los Estados Unidos o de polvo transportado por tormentas de riento desencadenadas en Africa.

Se explica así la preocupación por el deterioro del medio humano. Esta preocupación se manifiesta en eventos académicos, como la conferencia celebrada durante 1970 en el Williams College, que dejó como resultado un voluminoso e influyente informet. Desde un punto de vista práctico, se manifiesta también en el hecho de que los paises industrializados estén promulgando una legislación cada vez más frondosa para la protección de la ecología. Las políticas $y^{r}$ actividades desarrolladas en los Estados Unidos hasta 1971 se encuentran resumidas en el segundo informe anual del Council on Environmental quality (agosto de 1971). Del mismo modo, en Japón se han dictado recientemente numerosas leyes sobre la materia y se ha establecido una agencia que dispone ingentes recursos. En 1971, la Comunidad Económica Europea adoptó un plan de acción cuyos principales lineamientos consisten en el establecimiento de una política común, que deberia confiarse a una agencia comunitaria, en la adopción de las medidas de control y en el uso de instrumentos fiscales para reducir la contaminación. Complementando estos esfuerzos se espera que la Conferencia de Estocolmo redunde en la aprobación de un Plan de Acción, de un Fondo y de una Agencia destinados a luchar contra estos problemas a escala mundial.

¿HACIA LA PARALJZACIÓN VOLUNTARIA DEL CREGMMMENTO ECONÓMICO?

Este tipo de preocupaciones es el que ha conducido, en los paises industrializados, a la sorprendente tesis de que ha llegado el mo-

'Man's Impact on the Global Entronment: Assessment and Recommendation for Action, MIT Press, Cambridge, Mass, 1970. 
mento cle detener el crecimiento. La tesis del zero grozulh, anticipada con escándalo por unos cuantos economistas heterodoxos, ya ha saltado a la primera página cle los periódicos más influyentes sle Estados Unidos y Europa, en los cuales en seguida adquirió tintes alarmistas. La idea clesafía todas las nociones generalmente aceptadas. El bienestar de la sociedad se mide por el tamaño dei proclucto nacional bruto. El PNB es un concepto susceptible de medición estadística, por lo que satisface las exigencias de una concepción tecnocrática de la sociedad contemporánea, $y^{\prime}$ aparentemente incluy'e todos los bienes $y^{\prime}$ serricios que genera la comminidaci en el transcurso del tiempo para satisfacción de sus miembros. La mayor parte de la gente está convencida de que su bienestar depende cle una mayor disponibilidad de automóviles, televisores, refrigerado. ras, lavadoras, viajes y peliculas y otras ventajas cuya producción incide fuertemente en el crecimiento del producto. Pero pocos advierten que el mismo proceso que genera esas comodidades es responsable de que las ciudades se estén convirtiendo en gigantescas usinas de acero y de cemento, de la pesadilla clel tráfico motorizado, la destrucción del paisaje $y^{r}$ los recursos naturales, y la alienación del indiriduo frente a la organización social $y$ a los medios de comunicación cle masas. La comprobación de estos hechos está llevando a pensar en la necesidad de regular el crecimiento econó mico e incluso de transferir una parte de los recursos que actualmente se aplican a la aceleración de ese proceso hacia la adopción de medidas encaminadas a mejorar la calidad de la vida $y$ transformar el medio físico, social y cultural del hombre."

Paradojalmente, estas tendencias se encuentran con posturas hasta cierto punto coincidentes en los países en desarrollo. Aparte de que estos países ostentan una tradicional preferencia por los valores humanísticos -responsable en buena medida de su falta de desenvolvimiento económico- ahora empicza a despuntar en ellos la crítica al PNB como expresión de desarrollo descle el propio punto de vista de las políticas económicas. En efecto, hoy resulta claro que en muchos de estos países el crecimiento económico se ha desarrollado de tal manera que sus frutos se han reparticlo en forma muy desigual, creando agudas tensiones que perturban la paz del globo. Surge así la idea de buscar un modelo de desarrollo más concordante con la dotación de factores con que cuentan esos países y más orientado a la satisfacción de las necesidades básicas de las mayorías de sus poblaciones. El énfasis unilateral en el ta-

VVer el documento preparado por Ignacy Sachs para el Secretariado de UNESCO, Environmental Quality Mangement and Development Planning, Ginebra, 1971 . 
maño del producto ha sido seguido de una mayor preocupación por su composición, por las técnicas elegidas para generarlo y por la distribución de los bienes que lo componen. Se empiezan a analizar con cuidado algunas experiencias que muestran que una tasa elevada y hasta espectacular de crecimiento del PNB pueden ir acompañadas de un desempleo generalizado y de una distribución regresiva del ingreso. Se comienza a pensar en modelos basados en la movilización de las fuerzas de trabajo disponible, en la aplicación de tecnologías intermedias, en un patrón de consumo más igualitario y cn un tipo de producción que sirva para llenar una "canasta" de bienes esenciales, con un mejor equilibrio entre la industria y la agricultura. $Y$ no se trata de preocupaciones conlinadas a pequeños grupos disidentes, sino de opiniones originadas en el sancla sanctorum del desarrollo: nada menos que el Banco Mundial. El señor Mahabub ul Haq, distinguido, economista y asesor del presidente del Banco, dirigiéndose a la 12a Conferencia Mundial de la Sociedad para el Desarrollo Internacional celebrada en la ciudad de Ottawa, scñaló la necesidad de colocar el desarrollo "sobre sus pies" y para ilustrar esta idea se refirió a la experiencia china, mientras que el propio Mc Namara, al hablar ante la UNCTAD III, criticó los modelos de desarrollo que dan lugar a una mayor concentración del ingreso, citando cifras formadas de las experiencias de Brasil y México. ${ }^{6}$

Es cierto que estas tendencias todavía distan mucho de haber ganado aceptación universal. En los países avanzados, la lucha por la calidad de la vida cuenta con el apoyo entusiasta de los grupos más acomodados ${ }^{\top}$ y con el de los hijos de esas personas, los cuales militan en la "nueva izquierda" u otros movimientos radicales, pero no ha podido atraer a los "blue collars" y a los peldaños más bajos de las sociedades respectivas, para quienes sigue siendo más importante mejorar su nivel de vida. En los países en desarrollo, esta tendencia a cuestionar la validez del slogan del crecimiento uber alles choca con la indiferencia de la ortodoxia económica y con ataques provenientes de aquellos sectores marxistas que postulan la necesidad de un proceso "staliniano" -basado en la acu-

'Ver Employment in the 1970's: A New Perspective, documento presentado por el señor MLahabub ul Haq en la XII Conferencia Mfundial de la Sociedad para el Desarrollo Internacional, y' cl Discurso ante la Conferencia de las Naciones Unidas sobre Comercio y' Désarrollo dèl presidente del Banco Mundial, señor Rolbert S. Mc Namara.

${ }^{7} \mathrm{La}$ Conferencia de las Naciones Unidas sobre el Medio Humano atrajo a Estocolmo una multitud de mús de diez mil conservacionistas - coleccionistas botánicos, amigos de los animales, bird watchers y otros aficionados cuyos intereses no tienen mucho en común con los grupos más pobres de la sociedad. Ver Time, 22 de mayo de 1972. 
mulación a expensas del consumo y en la construcción a marchas forzadas de una base industrial- o a la estrategia alternativa, en ausencia de una revolución, de estimular al capitalismo para que cumpla esta tarea cuyos frutos heredará el estado socialista una vez que el régimen capitalista se haya derrumbado por obra de las contradicciones inherentes a ese modelo de desarrollo.

Én todo caso, estas tendencias existen tanto en los países ricos como en los subdesarrollados. Aunque en uno y otro caso difieren en sus motivaciones y resultados, parecen coincidir en que ambas dan aliento a una especie de rebelión-contra el absolutismo del PNB como expresión de bienestar y medida del desarrollo.

Desde un punto de vista conceptual, hoy tiende a reconocerse con mayor claridad que, aunque fácilmente cuantificable y por lo mismo conveniente para fines operativos, la noción del PNB resulta, por numerosas razones, un concepto demasiado estrecho. En primer lugar, se presume que el desarrollo tiene que ver solamente con los flujos de ingreso que genera una economia y no con los inventarios previamente acumulados, tanto físicos como sociales, de los cuales depende en gran medida el bienestar de la comunidad ${ }^{8}$. En segundo lugar, este enfoque cuantitativo supone que una serie de valores relacionados con el cambio social, el desarrollo cultural y la calidad de la vida, carecen de significación económica, así como tampoco pesan los efectos negativos vinculados a los costos sociales de determinadas actividades, por la sencilla razón de que, de conformidad con los conceptos económicos imperantes, ni unos ni otros se contabilizan en el cálculo del producto nacional bruto. En tercer lugar, se ignora que teóricamente el producto constituye un concepto ambiguo y de muy dificil comparación entre los distintos países, ya que necesariamente consiste en una colección de bienes -como zapatos, libros y automóviles- para cuya tasación es necesario aplicar un sistema de pesos y medidas (el llamado sistema de precios) esencialmente relativo, puesto que depende de la dotación de factores y de los costos comparativos prevalecientes en cada economía. En cuarto lugar, también se tiende a olvidar las dificultades que se presentan cuando se trata de medir las modificaciones del PNB durante largos períodos de tiempo, como consecuencia

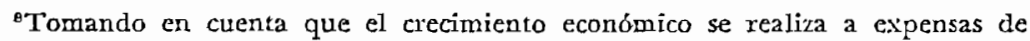
las reservas de rccursos no renovables y de la capacidad del ecosistema para absorber la contaminación $\rightarrow$ dos factores intrínsecamente limitados- Kenneth Boulding ha propucsto que el PNB considere el "costo nacional bruto", equivalente a la destrucción de los correspondientes inventarios. Ver K. Boulding, Environment and Economics, en W. W. Murdoch, Environment, Sinauer Associates, Stanford, Conn., 1971. 
de cambios en la población, en sus preferencias, en la composición del producto o en las relaciones de precios.

Desde otro punto de vista, el excesivo énfasis en el PNB como medida del desarrollo, a expensas de los aspectos vinculados a la calidad de la vida, se basa en la presunción de que ningún grado de deterioro experimentado en este último plano podría llegar a comprometer el proceso de inversión y de crecimiento del producto, lo que equivale a olvidar que el hombre es el motor de todo sistema económico y que cualquier circunstancia que afecte su bienestar y su desarrollo personal repercutirá fatalmente en la capacidad de sistema.

No se trata de argumentar que el crecimiento del producto no: constituye un objetivo importante del proceso de desarrollo sino de sostener que el PNB no debería usarse como único indicador. del. bienestar de la comunidad. En suma, el PNB es un concepto que: excluye un gran número de factores que, en la práctica, contribuyen positiva o negativamente a determinar el nivel de bienestar.. Así, por ejemplo, no se incluyen en el PNB una serie de deseconomías externas, tales como el humo y los ruidos, que se traducen en: el deterioro del medio físico o del ambiente psicosocial. Tampoço se incluyen la mayor parte de los aspectos cualitativos de los bienes y servicios de que dispone la comunidad, tales como la belleza del paisaje o la calidad cle un programa de radio o televisión. Otros factores son excluidos como consecuencia de la organización social del trabajo y del sistema de precios prevalecientes en la sociedad: el mero hecho de que en las sociedades avanzadas la ropa se :lave a máquina y el cuidado de los ancianos se encuentre institucionalizado determina que estas actividades se reflejen en el PNB, a diferencia de lo que ocurre en los paises subdesarrollados. En cambio, el cálculo del producto se acrece por obra de una serie de gastos compulsivos que la gente preferiría no efectuar, como aquellos que realizan los paises en actividades bélicas o por razones de prestigio internacional.

La ecología forma parte de esta nueva preocupación por la calidad de la vida y el interés que clla despierta alimenta la tendencia al cuestionamiento del PNB como medida del desarrollo. Si bien es cierto que estas inquietudes no están en la mente de todos los defensores del equilibrio ecológico, resulta paradojal que sólo como un intento de salvaguardar el marco físico en que se desenvuelve la humanidad, haya tenido lugar una nueva apreciación de los valores no materiales de la vida. "Cualquier actividad humana que no requiera un gran flujo de recursos no renovables o no prodúzca una profunda degradación ambiental puede continuar creciendo indefinidamente. En particular, podrán florecer ạquellos objetivos que 
mucha gente contaria entre las actividades más deseables y satisfactorias del hombre - la educación, el arte, la música, la religión, la 'investigación científica, la cultura física y la interacción social". 9

Pero también hay mucho de negativo o, al menos, de cuestionable en este tipo de preocupaciones, en la medida en que abren paso a una postura que preconiza la congelación del status quo. Para empezar, la tesis resulta discutible en el ámbito de los propios países industriales, donde se ha originado. Al respecto, se plantean de inmediato dos interrogantes: ¿cuál es la racionalidad de una tasa de crecimiento igual a cero y cuáles son los grupos que, si el crecimiento se detuviera en su nivel actual, resultarían más perjudicados?

¿Por qué una tasa de crecimiento igual a cero? ¿Fue acaso planeado el nivel que hoy alcanza el producto interno bruto de los paises industrializados? ¿Hay algo de mágico en lo que respecta a los Estados Unidos por ejemplo, en un producto nacional cuya magnitud se expresa en trece cifras? Nadie podria pretender seriamente que la mano invisible que ha venido impulsando el desarrollo en los países avanzados, desde la revolución industrial hasta nuestros días, ha conducido a una especie de óptimo expresado en el volumen y composición que en 1972 exhilse el PNB de esas naciones. ¿Por qué, si el desarrollo está empezando a ser nocivo en las naciones ricas, no cambiarlo por un poco de subdesarrollo? ¿Por qué una tasa de crecimiento igual a cero $y$ no una tasa negativa, digamos, de -5 por ciento anual?

No hay ninguna razón, en efecto, para suponer que al asignar al rubro automóvil 19 centáos de cada dólar destinado al consumo, los norteamericanos han Ilegado a una distribución ideal de sus presupuestos personales o que ello sea preferible a un consumo menor de automóviles acompañado de mejores sistemas de transporte colectivo. No parece haber mucha racionalidad en el hecho de que de los 21 mil millones de dólares gastados en 1969 por el sector público en transportes, 18 mil millones se hayan destinado a calles y carreteras, mientras que sólo se invirtieron 358 miliones en transportes colectivos y 21 millones en lerrocarriles. Si se considera que la industria automotriz representa el $7,5 \%$ del producto y el $31,5 \%$ del consumo total en los Estados Unidos, se llega fácilmente a la conclusión de que, una vez que estos "óptimos" han sido puescos en tela de juicio, se torna aceptable la idea de que una reduc,ción del PNB podria resultar ventajosa para el bienestar de todos. ${ }^{10}$

${ }^{9}$ The limits to Growlh, op. cit., p. 175.

${ }^{10}$ Ver Wilfred Owen, citado en Environmental Costs and Priorities, documento -preparado por la Secretaría de la Conferencia de las N'aciones Unidas sobre el Medio Fumano para la rcunión de expertos efectuada en Founex, junio de 1971. 
Como ya se habia anticipado, también resulta sospechoso el hecho de que la causa del equilibrio ecológico haya ganado popularidad entre los grupos de mayores ingresos en los países industrializados. Lo que ocurre es que el crecimiento económico terminó por interferir en los privilegios de esos grupos. Los progresos en la agricultura acabaron con sus casas de campo, el mejoramiento dé: la productividad de la fuerza de trabajo determinó la desaparición del servicio doméstico, las carreteras se llenaron con los autos de "los demás" y los centros de turismo se volvieron masivos y vulgares. Resulta fácil disfrazar la defenșa de estos intereses detrás dẹ la fachada de una revuelta contra el endiosamiento del PNB y de la búsqueda de valores más auténticos y espirituales. Sin embargo, la comida, la vivienda y las condiciones de trabajo siguer siendo más importantes para una parte de la población de los pafses industrializados - sin decir nada por el momento acerca de los paises subdesarrollados- que la pureza del aire o la preservación del paisaje. Estas últimas aspiraciones no cobran verdadera vigencia mientras las necesidades básicas no hay'an sido satisfechas. Por lo tanto, es muy posible que cualquier cambio masivo en la asignación de recursos para la ejecución de programas de mejoramiento ambiental, implique una redistribución en favor de los miembros más ricos de la comunidad de esos países. Por último, uno de los niedios de combatir la contaminación consiste en aplicar impuestos a los agentes contaminantes, impuestos que son transferidos al común de la población a través de aumentos de precios, con su conocido efecto regresivo.

\section{EL MEDIO hUMANO Y EL DESARROLLO}

Pero las consecuencias más negativas de las tesis que propician la detención del crecimiento se perciben en relación con los países subdesarrollados.

Por de pronto, si bien estos planteamientos no preconizan la paralización del desarrollo en los países del Tercer Mundo, postulan en cambio la congelación de la distancia que existe actualmente entre ellos y los grandes centros inclustriales. "La primera prioridad de los paises ricos -sostiene uno de los documentos preparados por el Club de Roma- deberá ser, detener el crecimiento de capital; la de los paises pobres cleberá ser, detener el crecimiento demográfico

Ver también John Hardesty et al., Political Economy and Envitonmental Destruction, en el número de otoño de 1971 del Joumal of Radical Political Economy. 
Norobstante, esto no quiere clecir que en los primeros la población puedá aumentar sin control, y en los últimos que el crecimiento del capital pueda mantenerse indefinidamente... El crecimiento del capital en los países pobres, aunque debe permitirse que continúe por algún tiempo, tendrá que ser regulado de manera de minimizar el consumo de recursos y la generación de contaminación". El documento concluye afirmando que "no existe ninguna posibilidad de que la vasta mayoría de los habitantes de los países en desarrollo lleguen a alcanzar los niveles materiales de vida que disfrutan los países desarrollados".

Además, la detención del crecimiento en los paises industrializaclos no bastaría para evitar la destrucción ecológica a escala mundial. Podria pensarse que, con una tasa de crecimiento igual a cero los Estados Unidos estaríán cumpliendo su deber en esta materia. Pero no es así. Los Estados Unidos consumen una cuota de minerales, combustibles fósiles y otros recursos naturales muy superior a la que les corresponde. Poseyendo el 6 por ciento de la población mundial, los Estados Unidos emplean entre el 35 y el 50 por ciento de los recursos naturales que se extraen anualmente en el mundo, $y^{\prime}$ se estima que su consumo alcanzará entre el 60 y' el 80 por ciento del consumo mundial.11

Por último, la concepción que los paises inclustrializados poseen acerca de los problemas ecológicos no refleja las preocupaciones de los países subdesarrollados. En los primeros, estos problemas se producen como resultado de un largo proceso de acumulación de capital, de un grado muy avanzado de desarrollo tecnológico y de la presencia de elevados niveles de consumo. De allí que estos países asignen gran importancia a los efectos depredatorios de determinadas actividades principales responsables de la contaminación ambientạl y del rápido agotamiento de los recursos no renovables, sin prestar mayor atención a fenómenos vinculados a niveles muy bajos de desarrollo que asimismo conspiran contra lá calidad del medio humano.

Los países en desarrollo, en cambio, perciben los problemas ecológicos en forma diferente. Los suyos son problemas que tienen su explicación en la pobreza y en la falta de desarrollo de sus sociẹdades. En los páses industrializados, la alimentación representa el $18 \%$ de los presupuestos familiares, en comparación con porcentajes cercanos al $80 \%$ en el caso de los países de Africa y del sureste de Asia, por ejemplo. Las economías de los países indus.

MEstimación efectuada por Heather Dean, de Resources for the Future, en The Dynamics of American Imperialism, Radical Education Project, Ann Arbor, Michigan, 1971. 
trializados presentan una alta densidad cle capital, por contraste con las de los paises en clesarrollo, en clonde la mano de obra constituye el recurso más abundante. Estas diferencias en la estructura del consumo $y$ en la combinación de los factores productivos determina que los problemas ambientales que confrontan ambas categorias de paises sean también muy diferentes. Estas diferencias corresponden a otras tantas etapas en el proceso de desarrollo. Conforme la industrialización avanza, la tecnología se vuelve más compleja, la urbanización se expancle y' el consumo comprende una mayor proporción de bienes durables, las perturbaciones ambientales ocasionadas por la pobreza clisminuyen, y comienzan a advertirse los efectos nocivos clel crecimiento económico sobre la ecología. La tuberculosis o el tilus son recmplazaclos por el enfisema y el infarto. El clesequilibrio de la alsundancia sucecle al clesequilibrio de la pobreza.

Mientras estas etapas no se cumplan, la prcocupación por el medio ambiente en los países menos desarrollados seguirá siendo un aspecto esencial de su preocupación por el ciesarrollo. La comida, la vivienda, la salud y' las condiciones de vida y de trabajo seguirán siendo más importantes para la abrumadora mayoria de la población de esos países que la purcza del aire o la prescrvación del paisaje. listas últinas aspiraciones no cobran verdadera urgencia mientras las necesidades básicas cle la población no han sido satisfechas. In suma, pues, la mayor parte de los problemas ambientales que experimentan estos paises son básicamente cl resultado de un clesarrollo insufficiente: la ciesnutuición y la en[ermedad, las malas condiciones de vivienda y saneamicnto, el empobrecimiento y destrucción de sus recursos naturales por falta de medios para hacer posible la conservación y el mejor uso de los mismos.

En otras palabras, existen clos maneras en que los problemas ccológricos pueden amenazar la caliclacl, c incluso la posibilidad, de la vida humana. La primera se expresa en ciertas cxternalidades del proceso de industrialización y del progreso tecnológico que conducen a la destrucción o deterioro clel medio ambiente. La segunda, consiste en lat existencin cle agudos déficits relativos a las condiciones mínimats que debc remir el habitat humano, así como las que se requieren para la adecuada conservación y la plena utilización cle los recursos naturales. Podría clecirse que, mientras que el $90 \%$ de las preocupaciones de los países industrializados se refieren a esás externalidades y sólo el $10 \%$, a los déficits, todo lo contrario ocurre en los países del Tercer Nundo que se debaten en niveles muy bajos de desarrollo, sin haber alcanzado lodavía la etapa de recordar con nostalgia la era bucólica del "buen salvajc'.12

:Para uni versión extrema de esta tesis, en buena medicla coincidentc con la posición oficial de Brasil, ver M. Ozorio de Almeyda, Economic Development 
El primer borrador del informe redactado colectivamente por el panel de expertos convocado por el Secretario General de la Conferencia de las Naciones Unidas sobre el medio humano en Founex, Suiza, en 1971, rezaba en sus primeros púrralos:

1. La nueva preocupación sobre el medio ambiente ha atraido la atención hacia el hecho de que el desarrollo humano, hasta ahora, ha siclo alcanzado a costa del deterioro del medio humano.

2. Las politicas del futuro deberfan interesarse no solamente en reparar algo del daño ya causado, sino también en asegurar que el desarrollo futuro produzca tan poco daño como sca posible en el medio humano.

3. Todo esto implica que, ceteris paribus, el desarrollo futuro podria muy bien llerarse a cabo con costos unitarios más altos que cn el pasado.

4. El principal problema que se plantea entonces es cómo deberían compartirse estos costos adicionales entre los países desarrollados y los paises en desarrollo.

La clistorsión de este enfoque fue gradualmente advertida en el curso del mismo panel. Las conclusiones preliminares que se acaban de citar fueron sustituidas finalmentc, on el informe definitivo, por conceptos como los siguientes:

Puede afirmarse que, en gran medida, el actual interés en las cuestiones relacionadas con el medio ambiente ha tenido su origen en los problemas experimentados por los paises industrialmente adelantados. Estos problemas son cle por si, en gran parte, el resultado de un nivel elevado de dcsarrollo económico. La creación de una gran capaciclad de producción en la industria y en la agricultura, el crecimiento de sistemas complejos de transporte y comunicaciones, la rápida evolución de los conglonerados urbanos, son factores que, de un modo u otro, han causado daños y perturbaciones en cl medio humano. Esas perturba-

and the Environment: False Dilema, en Enviroment and Development, Carnegie Endowment for International Peace, 1971.

Una demostración de lo dicho se relleja en la mancra como sc organizaron los trabajos en la Conferencia de Estocolmo. El primer comite fuc asignaclo a tratar los problemas relacionados con los ascrtamientos humanos y con la educación y la cultura; el segundo comité trató la utilización de recursos naturales y las relaciones entre medio humano y desarrollo; la tercera comisión, el control de agentes contaminantes, las implicacioncs institucionales de la Conferencia y el programa de acción. Los aspectos prúcticos de la Conferencia queclaron vinculaclus estrechamente a los problemas de la contaminación. 
ciones han llegado a alcanzar tales proporciones que en muchos sitios constituyen ya un grave peligro para la salud y el bienestar humanos. En realidad, en ciertos aspectos los peligros rebasan las fronteras nacionales y amenazan a la totalidad del mundo... Sin embargo, los principales problemas ambientales de los países en desarrollo son básicamente diferentes de los que se perciben en los países industrializados. Son principalmente problemas que tienen su raiz en la pobreza y la propia falta de desarrollo de sus sociedades. En otras palabras, son problemas de pobreza rural y urbana. Tanto en las ciudades como en el medio rural lo que está en peligro no es solamente la "calidad de la vida", sino la propia vida, debido a deficiencias en el abastecimiento de agua, la rivienda inadecuada, la falta de higiene $y$ la baja nutrición, las enfermedades y las catástrofes naturales. Son estos problemas los que, en grado no menor que la contaminación producida por la industria, exigen atención en el contexto de la, preocupación por el medio humano. Son, por lo demás, problemas que afectan a la parte más numerosa de la humanidad. ${ }^{13}$

Mientras no haya una clara comprensión acerca de estas diferencias básicas los países industrializados no presentarán atención a los problemas ambientales del Tercer Mundo y, lo que es peor, continuarán interfiriendo en las decisiones ecológicas de estos últimos en función de criterios equivocados. Es lo que ocurre cuando la opinión pública de los paises industriales, o algunos personajes. supuestamente bien intencionados, tratan de influir a través de sus. gobiernos sobre los países en desarrollo para que éstos adopten decisiones coherentes con sus propias ideas, sin tomar en cuenta los. intereses locales. Tal fue el caso, por ejemplo, del éxilo que apa. rentemente tuvieron algunos científicos británicos en los esfuerzos desplegados para que su gobierno se desistiera de financiar una parte de la construcción de una represa en las Cataratas de Murchison, en Uganda ${ }^{14}$. Los mismos prejuicios respaldan la propuestat formulada por el ex embajador George F. Kennan en la edición: de Foreign Affairs de abril de 1970, para el establecimiento de una agencia mundial de carácter independiente encargada de la conservación y protección del medio ambiente. Estas funciones no podrían ser cumplidas en forma adecuada por los organismos internacionales ya existentes los cuales por lo general abordan los pro-

rasl Desarrollo y el Medio (tema V), documento de la Confcrencia de las Naciones Unidas sobre el medio Humano, Anexo I, pp. 1 g' 2.

${ }^{34}$ Paul Geroudct, "Arurchison Falls en Ouganda: Destruction on protection",. Biological Conservation, julio de 1970. 
blemas de su competencia desde el punto de vista de los objetivos del desarrollo, pues a juicio del embajador "las consideraciones desarrollistas con frecuencia están en conflicto con las necesidades relativas a la conservación del ambiente". Especialmente ilustrativos son aquellos aspectos en que el autor se refiere al control de la agencia propuesta.

Buscar la aprobación de toda la comunidad internacional para la creación y funcionamiento [de esta agencia] no constituiria un esfuerzo muy promisor. Dejando a un lado el hecho de que ello requeriria procedimientos que en la práctica no podrían distinguirse de los de las Naciones Unidas, esto significaria dar participación en el control y la operación del nuevo ente a una multitud de países poco desarrollados y pequeños que podrían contribuir muy poco a la solución de los problemas planteados. Ello envolveria, al mismo tiempo, formidables demoras y graves problemas para la adopción de decisiones... Se llega así a la conclusión de que si algo constructivo pudiera ser realizado a lo largo de estos lineamientos, el interés y la iniciativa deben proceder de un grupo relativamente pequeño de gobiernos; y la lógica sugiere que aquellos gobiernos deben ser los de las grandes naciones industriales y' marítimas. Son ellos los países cuyas economías producen la mayor parte de los problemas relacionados con la contaminación. Son ellos, de nuevo, los que disponen, los que tienen los recursos cientílicos y de otro orden para analizar los problemas e identificar las líneas de solución más promisoras. .5

Resulta fácil imaginar las desastrosas consecuencias que para los países subdesarrollados traeria la institucionalización de las falacias que se discuten más arriba a través de una agencia como la propuesta por el señor Kennan.

Estas diferencias en cuanto a la manera como son percibidos los problemas ecológicos tienden a refiejarse en la forma de abor-dar sus soluciones. Es probable que los países menos desarrollados no pongan tanto énfasis como las naciones avanzadas en la adopción de medidas correctivas, que tenderían a regular el crecimiento inclustrial $\mathrm{y}$ que eventualmente podrían imponer un ritmo más lento al proceso de desarrollo. Es importante, en efecto, distinguir aquellas medidas encaminadas al mejoramiento ambiental que tien- den a frenar el crecimiento económico de aquellas que no lo afec-

${ }^{15}$ To Prevent a World Wasteland: A Proposal, por Gcorge F. Kennan, Foreign Affairs, abril de 1970, p. 410. 
tan en forma negativa. Las políticas ecológicas en los países avanzados suponen la realización de inversiones adicionales para paliar los efectos destructivos de las inversiones ya efectuadas. En los países subdesarrollados, en cambio, el problema se plantea en términos de obtener una mejor asignación de los recursos disponibles. De aquí se siguen varias consecuencias.

- El énfasis en las consecuencias negativas de un desarrollo excesivo conduce al diseño de políticas ecológicas basadas en elementos de carácter tecnológico. El reconocimiento de la contribución positiva que puede efectuar el desarrollo al mejoramiento del medio humano conduce a la formulación de políticas de contenido marcadamente económico y social: Ahora bien, es preciso precaverse en contra de la ilusión de que en los países pobres es posible mejorar el medio humano sin enfrentar los problemas de la cistribución del ingreso, el desempleo y el cambio social.

- Las políticas ambientales basadas en el enfoque estrecho de los paises industrializados tienden, por definición, a imponer costos adicionales sobre los proyectos de inversión que pudieran resultar en el deterioro del medio ambiente. Obviamente, sería muy difícil que los países en desarrollo adoptaran políticas excesiramente costosas. La solución de las deficiencias que afectan las condiciones de vida en áreas rurales y urbanas y la implantación de medidas para la conservación $y^{\prime}$ mejor uso de los recursos naturales dan Iugar, en cambio, a proyectos que forman parte del proceso normal de inversiones de un país y que, en general, no implican costos adicionales.

- Mientras que las medidas diseñadas en los países avanzados para la preservación del medio suelen requerir tecnologías muy sofisticadas y el empleo intensivo de capital, la mayoría de los proyectos orientados hacia la solución de los problemas que afectan el medio humano en los países subdesarrollados - vivienda, transportes, agua potable, forestacion- supone un uso intensivo de mano de obra. En estos países existe, como se señala más adelante, una intima relación entre la preocupación por el medio y el infasis en el empleo.

- Si bien los efectos destructivos del desenvolvimiento indus. trial y tecnológico también se dejan sentir en los países en desarrollo, existen aquí más posibilidades de que la solución de estos problemas no quede confiada exclusivamente a la adopción de medidas correctivas o preventivas, que inevitablemente encarecen el costo de los proy'ectos o se traducen 
ESTUDIOS INTERNACIONALES

en nuevas inversiones. En los países del Tercer Mundo, gran parte de esos efectos podrian evitarse mediante el desarrollo de un patrón de consumo y un tipo de producción más a tono con sus valores culturales y con los niveles de ingreso de la mayoria de la población, en lugar de imitar indiscriminadamente el modelo de las naciones industrializadas, lo que podria contribuir a limitar el número de automóviles, la magnitud de los conglomerados urbanos o el volumen de desperdicios no degradables, para citar tan sólo unos cuantos ejemplos.

Pero el hecho de que en estos paises los esfuerzos para mejorar el medio humano sean una parte integrante de su lucha por el des. arrollo no debe llevar a la conclusión de que los problemas ambientales se identifican sin más con los de la pobreza ni a creer que el proceso de desarrollo tenderá a solucionarlos en forma automática. Se trata de problemas que deben ser percibidos en forma dinámica y que cambian a través del tiempo conforme el propio proceso de desarrollo va dando lugar a efectos negativos no deliberados. Es evidente que este tipo de problemas no puede resolverse espontáneamente mediante la mera aceleración del crecimiento económico. En algunos casos, nos encontramos frente a problemas de arrastre que no fueron abordados, como la erosión del suelo o la urbanización no planificada. En otros, tenemos que enfrentar las consecuencias imprevistas de determinadas inversiones, como los cambios climáticos $y$ la difusión de enfermedades epidémicas derivadas de la construcción de gxandes embalses y represas. Por lo tanto, también en estos países la preocupación por el medio humano debería servir para imprimir nuevas dimensiones al concepto mismo de desarrolio. En tal sentido, las políticas ecológicas deberían ser parte integrante de la política general de desarrollo e incorporarse en forma explicita en el proceso de planificación económica $y$ social. En otras palabras, el mejoramiento ecológico debería considerarse como uno de los objetivos del plan. Este objetivo comprendería aspectos referentes al empleo y al ingreso, a la alimentación, la salud, la vivienda, la educación y el desarrollo de la comunidad.

Pero las implicaciones más importantes de esta creciente preocupación por el equilibrio ecológico mundial no se plantea con respeclo a las politicas internas de los paises en desarrollo sino a. sus relaciones económicas con las naciones industrializadas.

Existe el temor de que el malestar ecológico en los países avanzados afecte negativamente el comercio de los países en desarrollo, si la adopción de criterios ambientales más estrictos para el ingreso 
de productos provenientes del Tercer Mundo da Iugar a tendericias "neoproteccionistas" en los países avanzados y si el reaprovechamiento de las materias primas comienza a practicarse en gran escala. Existe tambien el temor de que la tecnología transferida por los paises avanzados se vuelva cada ve\% más cara y menos adecuada a las necesidades de los países del Tercer Munclo como resultado del desarrollo de dispositivos anticontaminantes, que encarecerian extraordinariamente el costo de los proyectos. Por último, existe el temor de que esta preocupación incluyca a los gobiernos y' al público de los paises industrializaclos a asignar cada vez más recursos a la lucha contra la cestrucción clel ambientc, con la consiguiente disminución en los niveles de la ayucla. La última parte de estás rotas están dedicadas a estos problemas.

\section{LA PREOCUPACIÓN ECOLÓGICA Y LOS PAISLS EN DESARROLI.O}

Algunas de las medidas relativas al medio humano que cstán comenzando a aplicar los paises industrializados afectan directamente a las relaciones económicas internacionales -especialmentc al comercio mundial- en desmedro de los países en clesarrollo. Pero en definitiva, todas las actividades que emprenclan las naciones más avanzadas en relación con la ecologia afectarán a los países subdesarrollados, al dar lugar a gastos aclicionales y a una reasignación de los recursos de que disponen las primeras, asi como al atterar la demanda y la estructura mundial de la producción y del comercio. Por un laclo, se desarrollarán nuevas tendencias, como ocurte con el consumo de materialcs y cquipos empleados en la lucha contra la contaminación. Según estimaciones de la revista Forlune (febrero de 1970) el mercado para estos productos en los Estados Unidos sobrepasa los 500 millones de dólares y estú creciendo a un ritmo clel $15 \%$ al $20 \%$ al año, mientras que el mercaclo de servicios para el mantenimiento de esos equipos sigue lincamientos semejantes. De acuerclo con Business Week (23 cic enero de 1971), lo mismo ocurre en Europa. Se cstá creando así una nucva corriente de comercio internacional. Por otra parte, el comercio mundial experimenta importantes rcajustes, en cietrimento de determinados productos o países, como ocurre con la disminución clel uso del carbón y' el aumento del uso del petrólco y el gas natural como fuentes de energia, y con la clisminución del consumo cle petróleo del Cercano Oriente, de alto contenido de azufre, en favor del petróleo norteamericano. ${ }^{10}$

\footnotetext{
${ }^{10}$ Repercusiones de lis Políticas Relativas al Medio Ambiente sobre cl Comercio y el Desarrollo, en particular de los Países en Desarrollo. In[orme de
} 
Ha de comenzar por advertirse que las implicaciones internacionales del temor a un colapso ecológico tienen sus aspectos positivos para los paises del Tercer Mundo: En primer lugar, esta preocupación puede ser utilizada por los países en desarrollo para ganar más apoyo para programas y proyectos correspondientes a los sectores sociales - dcsamollo rural y urbano, salud y alimentación, saneamiento ambiental y educación-. Tradicionalmente, la ayucla exterma ha tendido a alejarsc de tales proycctos, debido a su baja tasa de retorno, al menos en el corto plazo y de acuerdo con los criterios que actualmente se utilizan para analizar la relación costo-beneficio. En scgundo lugar, esta acticud de alarma podría dar lugar a una mejor distribución geográfica de las industrias a escalá internacional, ya que conforme se vayan clevando los costos ambientales en los paises avanzados podria resultar provechoso trasladar alguna de sus inclustrias o localizar sus planes de expansión en los países menos desarrollados. En tercer lugar, estas tendencias podrian fortalecer la posición de las materias primas provenientes de los países subdesamollados, cuya demanda podría aumentar como consecuencia del rechazo a un uso inmodelado de productos sintéticos no degradables ${ }^{17}$. En cuarto lugar, también podria alentarse un mayor grado de elaboración de las mismas materias primas -especialmente los minerales - en los paises productores antes de exportarlas a los grandes centros consumidores. Es útil recordar aqui que el tratamiento de los metales es una de las actividades industriales más contaminadas y cuyos costos ecológicos adicionales podrían resultar más altos. En quinto lugar, casi todos los programas internacionales que se diseñen para combatir la contaminación de los mares contribuirán a la conservación de los recursos pesqueros de los paises en desarrollo, e igual cosa ocurrirá con los programas encaminados a prevenir y controlar ciertos desastres naturales. Por último, es probable que la ayuda externa tienda a condicionarse a la adopción de ciertas medidas que requieran movilizar mano de obra redundantc, en obras de conservación del suelo y adecuado uso de la tierra, aplicación de fertilizantes y de pestjcidas, forestación y drenaje, o construcción de redes separadas de colectores de aguas pluviales y servidas en las ciudades. Los países en desarrollo deberían estar alcrtas para aprovechar estas oportunidades.

\footnotetext{
la Secretaria de la UNCPAD, tercer periodo de sesiones de la Conferencia de las Naciones Unidas sobre Comercio y ljesarrollo, 1972, pp. 5 y 6.

${ }^{17}$ En los Estados Unillos, entre 1902 y' 1968 el consurao de jabón disminuyó en 71 por ciento como consecuencia del consumo de detergentes sintéticos, y el consumo de algodón declino en 33 por ciento entre 1950 y 1968 debido a la competencia de las libras sintéticas.
} 
Pero estas oportunidades se comparan desfavorablemente con. los riesgos casi ciertos que amenazan a los palses subdesarrollados. como efecto de estas tendencias. En el plano comercial, existe el peligro de que las exportaciones de productos primarios sean desplazadas por el desarrollo de tecnologias no contaminantes, de que la demanda de otros productos se reduzca por el reaprovechamiento de las matcrias primas y' ce que los paises inciustrializados comiencen a protegerse detrás de barrerus no arancelarias en contra de los productos provenientes de los paises en desarrollo que no cumplan con estrictos criterios de calidad ambiental.

La inplantación de tecnologías no contaminantes constituye ya un proceso en marcha. Asi, por ejemplo, en los Estadlos Unidos al igual que en otros paises se están tomando medidas para reducir el empleo de plomo como aditivo en los carburantes de los automóviles. Entre 1968 y 1971 en Suecia se redujo en un $20 \%$ el contenido medio de plomo de los carburantes. Tales restricciones han contribuido al estancamiento por que atraviesan actualmente las exportaciones de ese metal desde los paises en desarrollo. Ya se ha hecho mención a los cambios ocurridos en el comercio mundial como consecuencia de la preferencia por el petróleo de bajo contenido. de azufre, $y$ la preocupación que existe en los paises avanzados en torno a los detergentes no degradables podría abatir las importaciones de las materias primas respectivas. También podrian encontrarse numerosos ejemplos de restricciones a la entrada de productos alimenticios - tales como rrutas y vegetales, carne y productos lácteos- en los mercados de los páses industriales.

El "reciclaje" de materias primas, esto es su reaprovechamiento en el ciclo productivo, podria llegar a causar un verdadero terremoto en las exportaciones del Tercer Mundo. Hasta el momento, esta idea ha confrontado serias dificultades económicas y tecnológicas, y su aplicación masiva no es previsible en el futuro inmediato. Sin embargo, las investigaciones encaninadas a viabilizarla podrian intensificarse si aumentaran los precios de los productos primarios, por lo que la sola presencia de esta alternativa tenderá a actuar como freno sobre dichos precios. Por otra partc, ya se ha puesto. en circulación la idea de implantar un impuesto a las materias primas, con miras a estimular su reaprovechamiento y a evitar su extinción ${ }^{18}$. En el largo plazo, existen grandes probabilidades de queel reaprovechamiento en gran escala de los recursos naturales vulnere sustancialmente las exportaciones de los páses subdesarrollados.

Ưn número cada vez más extenso de productos básicos se está ${ }^{2 B}$ Blueprint for strvival, op cit., p. 10. 
viendo afectado por reglamentaciones sanitarias dictadas por los paises industrializados que funcionan, directa o indirectamente, como barreras no arancelarias ${ }^{10}$. Por otra parte, la elevación de los costos inclustriales como consecuencia de la adopción de tecnologias no contaminantes en los países avanzados tenderáa a traclucirse en sus precios internos, lo que según el documento de UNCTAD anteriormente citado daria lugar a un alza de entre el $4 \%$ y el $9 \%$ en los precios cle exportación que apliquen esos mismos paises. Esto último provocará fatalmente una tendencia hacia la protección de aquellas industrias cuyos costos y precios hay'an aumentado. Los países subdesarrollados, entre tanto, conseguirán lo peor de los dos mundos al verse obligados a importar más caro los productos de los paises industrializados $y$ a superar barreras arancelarias $y$ no arancelarias más elevaclas para ingresar a sus mercados.

El perjuicio causado a los páses en desarrollo por la aplicación -o con el pretexto- de políticas ambientales impuestas por los miembros más fuertes de la comunidad internacional podrian resultar muy graves si no se adoptaran oportunamente determinadas medidas. En primer lugar, los países industrializados deberian renunciar a la aplicación unilateral de normas ambientales; para que estas normas resulten internacionalmente aceptables deberían ser negociadas a cscala igualmente internacional. En scgundo lugar, y a los efectos anteriormente señalados, delsería establecerse un sistema mundial cle información $y$ de consultas previas en relación con las normas ambientales que los paises industrializados estén proyectando implantar. En tercer lugar, cada vez que sea posible, estas normas debcrian adaptarse a las condiciones de cada región, en lugar de ser aplicadas de una manera uniforme a escala mundial:0. En cuarto lugar, cuando la aplicación de estas normas afecte seriamente las exportaciones de decerminados paises en desarrollo, lá comunidad internacional clebería estar clispuesta a proporcionarles asistencia técnica y' financiera para ayudarlos a cumplir esos nucvos requerimientos e incluso compensaciones con el objeto de impedir que el desarrollo de los paises afectados se retrasc, en el corto plazo, como consecuencia de pérdidas por conceptos de exportaciones. Si no se acordaran mediclas tales como las señaladas, podría resultar muy fácil para los paises industrializados invocar políticas ambientales para amparar prúcticas comcrciales discliminatorias, reclucir el acceso de los productos provenientes de los países en

"Industrial pollulion control and foreign trade, documcnto preparado por la secretaría del GATT, Ginebra, 1971.

${ }^{20}$ Para un punto de vista opuesto, ver Pollution: Precedent and prospects, por . Charles Ch. Humpstonc, en Foreign Affairs, cnero de 1972, p. 332 y ss; 
desarrollo a sus propios mercados y fortalecer sus tendencias proteccionistas.

Otro plano en el cual la preocupación por el medio ambiente puede tener importantes repercusiones internacionales se refiere al tipo de tecnologia que Ios países industrializados transfieren al mundo en desarrollo. Se ha denunciado insistentemente que la tecnología que los paises industrializados exportan al Tercer Mundo supone un uso excesivo de capital y no está en concordancia con la combinación de factores productivos existentes en estos últimos países, en donde el recurso abundante es la fuerza de trabajo. Es muy probable que, en el futuro, la tecnologia desarrollada por los países avanzados sea aún más inapropiada para los países en desarrollo, al incorporar en forma masiva medidas no contaminantes en las nuevas plantas, equipos y procesos. La tecnología no contaminante del futuro, por innecesaria que sea para los países en desarrollo, les resultará más costosa. No existen estimaciones seguras sobre el costo adicional de esas tecnologías, pero se mencionan cifras que varian entre el $5 \%$ y el $20 \%$ de aumento. Un estudio reciente sobre los costos en que deberían incurrir las empresas cupríferas de los Estados Unidos si quisieran poner en práctica disposiciones contra la contaminación del aire por emisiones de anhidrido sulfuroso, llega a la conclusión de que las inversiones respectivas de. berian elevarse a 87 millones de dólares, según unos, y hasta 607 millones, según otros, lo que implica un costo adicional de entre el $5 \%$ y el $30 \%$ del costo actual por libra de cobre 21 . Leontief y Ford, por su parte, calcularon sobre la base de datos de insumoproducto los aumentos que se producirian en los precios de algunas de las principales industrias contaminantes de los Estados Unidos si sustituyeran combustibles de alto contenido de azufre por otros de bajo contenido, llegando a la conclusión de que las industrias químicas tendrían que aumentar sus precios en 10,3\%, las refinerías de petróleo en $8,3 \%$, las industrias de plásticos y sintéticos en $6,2 \%$, la minería del carbón en $6,1 \%$, y las industrias de abonos en $4,7 \%{ }^{2 *}$

Otro factor que podria afectar el desarrollo industrial en los países subdesarrollados es la previsible tendencia al desplazamiento internacional de inciustrias contaminantes por la emergencia de los llamados "pollution havens" en los países menos industrializados.

${ }^{31}$ Estados Unidos, Departamento del Interior, Bureau of Mines Information Circular/1971 No. 8527, Control of Sulphur Emissions in Copper, lead and zinc smelting.

"Leontief $y$ Ford, Air Pollution and the Economic structurc. Empirical results and input-output computations citado en Informe de la secretarfa de la UNCTAD, op. cit., p. 8. 
Estos desplazamientos ya se están produciendo en el interior de los propios paises avanzados. Así, por ejemplo, las nuevas normas sobre purificación del aire han llevado a reducir las operaciones de refinamiento de cobre en Arizona, Texas, Montana y Washington y a incrementar el embarque del mineral a refinerias localizadas en Alemania Occidental, Canadú $y^{r}$ Japón. Y este último país, a su vez, envía partidas de minerales para ser refinados en Indonesia. Como ya se ha señalado, los países en desarrollo podrían encontrar ventajas en una especialización en industrias contaminantes mutuamente convenida con los países industrializados. No habria nada de objetable en una política encaminada a atraer capitales foráneos hacia estas industrias, desplazadas de sus países de origen, toda vez que para los países del Tercer Mundo el problema se plantea en términos de cuánta contaminación adicional están dispuestos a absorber a cambio de más desarrollo. El peligro consiste en que estos desplazamientos no se traduzcan en un aumento real del flujo de recursos que se transfieren a los paises en desarrollo, sino que se limiten a operar un simple cambio en el contenido de un volumen fijo de inversión extranjera. En otras palabras, los países subdesarrollados deberian estar preparados para aceptar nuevas fuentes de contaminación siempre que ello vaya acompañado de inversiones adicionales y más altos niveles de desarrollo; de otra manera, lo que tendría lugar sería una mera sustitución de industrias no contaminantes por industrias contaminantes, sin aumento de las inversiones netas pero con niveles más elevados de degradación ambien. tal. En lugar de tener asi una mejor distribución mundial de las industrias se tendría solamente una nueva forma de especialización a escala internacional, en que las actividades contaminantes serian transleridas masivamente al mundo en desarrollo.

Por otra parte, debe considerarse que la radicación de industrias contaminantes tiene un costo implícito, que los países receptores deberían hacer valer, toda vez que la capacidad del ciclo natural para reabsorber deshechos tiene un limite y deberia ser considerada como nuevo recurso económico. Es cierto que los países subdesarrollados han utilizado menos sus recursos ambientales, lo que en el mediano plazo podría darles una rentaja comparativa para la instalación de estas industrias. Para ello seria necesario adoptar oportunamente decisiones de política en cuanto a la localización de las mismas, la adaptación de sus tecnologías al costo local de los factores y la eliminación de sus deshechos minimizando el daño del ambiente.

Los problemas relacionados con los costos de la tecnología y la orientación de las inversiones se plantean en forma crítica en la 
evaluación de projectos. Aquí tambièn existe el temor de que una excesiva preocupación por establecer normas ambientales, trasmitida a los países menos desarrollados a través de la asistencia técnica y de la cooperación internacional, pudiera distorsionar las prioriclades de inversión y los criterios de evaluación de proyectos adoptados por estos últimos. El problema se plantea en casi todos los sectores de la actividad económica. A título ilustrativo, se puede señalar que la lista de proyectos en los cuales deben tomarse en cuenta consideraciones ambientales, de acuerdo con la Agencia para el Desarrollo Internacional de los Estados Unidos, incluye actividades de construcción de carreteras, construcción de embalses, sistemas de irrigación, proyectos de agua potable y desagües, industrias petroquímicas y derivadas del petróleo, actividades mineras, desarrọIlo portuario, plantas generadoras de energia termales, hidroeléctricas $y^{\prime}$ nucleares, inclustria de la pulpa y el papel, aeropuertos, refinerias y plantas de fertilizantes. Se estima además que estas consideraciones deben intervenir en cada una de las etapas a través. de las cuales se desenvuelve un projecto: concepción de la idea, estudios de preinversión, ejecución de las obras y operación del. proyecto.

Para fines prácticos, en este plano se imponen algunas distinciones. Por una parte, es necesario distinguir aquellas consecuencias. ecológicas de un proyecto que tienden a obstaculizar el desarrollo. del mismo y' a reducir sus beneficios de aquellas no vinculadas al: éxito mismo del proyecto. Un ejemplo del primer caso lo proporciona nuevamente la gigantesca represa de Asuán. La desaparición de los depósitos anuales de limo en el valle del Nilo obligaron a construir plantas de fertilizantes artiliciales que utilizan una parte de la energía eléctrica generada por la represa. Por otra parte, tambien hay que distinguir entre las consecuencias ambientales que fueron previstas durante la formulación de un proyecto de aquellas que no lo fueron. Las primeras plantean el problema de programar las acciones que será necesario emprender a su clebido tiempo para paliar los efectos negativos previstos como resultado de la ejecución del proyecto, así como de identificar con la debida anticipación las posibles fuentes de recursos a que habrá de acudirse para financiar esas acciones. Las segundas plantean, en cambio, la necesidad de revisar las metodologías empleadas en la preparación de los proyectos $y$, eventualmente, de mejorar la información y la capacidad técnica disponibles. Por ejemplo, en los Estados Unidos, la Ley de Calidad Ambiental de 1969 dispone la evaluación de los probables efectos ambientales de cualquier proyecto financiado con fondos federales, antes de su ejecución.

En relación con los riesgos envueltos en el proceso de evalua- 
ción de proyectos para los países subdesarrollados, cabe formular tres observaciones. En primer lugar, está el peligro de que la excesiva preocupación por el sistema ecológico de los países industrializados los lleve a interferir en las decisiones soberanas de los países en desarrollo. Nadie discutiria, en principio, que las decisiones relacionadas con las prioridades del desarrollo y con los criterios aplicables en la evaluación de proyectos corresponden exclusivamente a los gobiemos respectivos. En la práctica, sin embargo, todos los días se registran violaciones a este principio. Los países subdesairollados no se encuentran obsesionados por el equilibrio ecológico y no debería imponćrseles criterios y costos adicionales, derivados de esa preocupación, que gravaran indebidamente su proceso de desarrollo. En segundo lugar, las deseconomías externas relacionadas con el medio ambiente son muy diffciles de prever y de cuantificar. En la medida en que estos criterios comiencen a intervenir en la evaluación de proyectos, tenderán a aparecer considerables diferencias de opinión entre unos y otros expertos, así como grandes discrepancias entre las consecuencias estimadas al formular el proyecto y sus efectos ex post. Esto podría conducir a aceptar los mayores costos sin una base de conocimiento adecuada. En tercer lugar, el análisis de los costos y beneficios sociales que debería aplicarse para estimar las secuelas ecológicas de un proyecto, no está suficientemente desarrollado. Este tipo de análisis incluye la medición de beneficios y costos secundarios y terciarios, que el análisis convencional generalmente no considera. Muchas veces, además, por su misma naturaleza estos costos y beneficios no pueden ser cuantificados dentro del marco del sistema de precios. Por último, también cambia el horizonte de tiempo contra el cual hay que calcular. la tasa de descuento del projecto. Por lo tanto, los países en desarrollo deberían abordar estas preocupaciones con extremada cautela.

De una u otra manera ya se ha hecho referencia al impacto que podría tener la preocupación por el medio ambiente sobre la ayuda internacional. Se teme que la alarma que existe frente a estos problemas en los países industrializados conduzca a una dism:nución de los recursos destinados a la ay'uda. Se estima que los Estados Unidos tendrán que asignar recursos del orden de los 20 mil y hasta los 30 mil millones de dólares para el cumplimiento de las normas ambientales ya aprobadas o pendientes de aproibación, lo que significa entre el $2 \%$ y el $3 \%$ del PNB de ese -país. Es evidente que estos gastos podrían distraer parte de los -j'a escasos recursos destinados a la ayuda externa, para asignarlos a la satisfacción de necesidades nacionales. Existe la posibilidad wle que los gobiernos y las agencias de cooperación internacional 
tiendan a imponer las normas ambientales adoptadas por los paísen avanzados, sin tener en cuenta la situación de los países en desarrollo. Como consecuencia de ello, podría ejercerse presión sobre los gobiernos de los paises subdesarrollados para ejecutar proyectos de baja prioridad destinados a mejorar el medio ambiente, los proyectos resultarían más caros, o podrían experimentar demoras en su tramitación debido al empleo de criterios de evaluación más complicados. $Y$ ciertamente los créditos atados servirían una vez más de caballo de Troya para introducir las tecnologías más costosas.

$*$

Lord Wayland Kennet, miembro de la Cámara de los Comunes por el partido laborista británico, sintetiza de la siguiente manera la argumentación de los países subdesarrollados:

No han transcurrido cincuenta años desde que Lugard avanzó la única defensa intelectualmente respetable del imperialismo que hasta ahora se ha intentado. En su libro Dual Mandate alegó que los poderes imperiales poseían -o debían poseerlos países coloniales a título de fideicomiso, por una parte, en beneficio de las propias poblaciones nativas y en tanto éstas aprendiesen a manejarse por si mismas $y$, por la otra, en beneficio de la humanidad en su conjunto, que precisa los minerales y cereales que los países retrasados todavia no necesitan. De ahi que sean ingratos Ios recuerdos que hoy despierta la. actitud de los países nórdicos al presentarse como portadores de una nueva lección, a saber, que los recursos que los paises sureños ahora necesitan $-\mathrm{y}$ ahora pueden explotar por sí mismos- todavia no pueden ser libremente explotados por temor a que el pupilo repita los errores que su maestro acaba de descubrir que cometió. Es como decir primeramente "ustedes no pueden tomar posesión de estas riquezas porque no lās necesitan y y'o si", para cleclarar cincuenta años más tarde: "ustedes todavía no pueden aprovechar esos recursos porque yo los usé en forma desmedida cuando tuve el control de ellos"..23

Lo interesante es que Lord Kennet, cuya fe en la santidad de la ecología incluso a expensas del desarrollo parece ser incon-

${ }^{29}$ Wayland Kennet, The Stockholm Conference in International Affairs, encro de 1972, pp. 39 y 40. 
movible, descarte esa argumentación calificándola de "una simple discusión entre hombres y hombres... superada por las dificultades entre el hombre y su ambiente; en otras palabras, entre una parte de la naturaleza y el todo".

La defensa de la ecología - para los que pueden gozar de ellano debe hacer olvidar las necesidades más apremiantes de los dos tercios más pobres de la humanidad. Los países en desarrollo deberian estar prevenidos contra los efectos adversos que podrian derivarse del malestar ecológico en los países avanzados. Y estos deberían abstenerse de presionar a los primeros para aceptar normas ambientales que no corresponden a sus propias ansiedades. Al fin y al cabo, los países industrializados saben que el efecto de demostración que ejercen sobre los paises pobres es muy poderoso, y habiendo capitalizado a menudo esa influencia en su propio beneficio, no deberían sorprenderse de que las poblaciones del mundo en desarrollo aspiren ahora a morir de contaminación, como los ricos. Por último, las grandes naciones no deberían olvidar que su propio y espectacular proceso de crecimiento económico se hizo a expensas de una explotación intensiva de la naturaleza, y no sería justa una posición que pretendiera impedir que los paises hoy subdesarrollados utilicen con liberalidad el ecosistema. El Tercer Mundo no debe soportar el precio que sería necesario pagar para hacer realidad el sueño de una tierra impoluta. 